\title{
Hábitos alimentarios en estudiantes \\ de medicina de primer y sexto año de \\ una universidad privada de Lima, Perú
}

\author{
Food habits in medical students in \\ first and sixth year of a private \\ university of Lima, Peru
}

\begin{abstract}
Objective: To assess food habits and factors associated with the consumption of soft drinks and fast food. Subjects and Methods: Cross-sectional study in first $(n=269)$ and sixth year $(n=165)$ students of the Faculty of Medicine at the University San Martin de Porres. A questionnaire structured was applied in two parts: the first part consisted of 11 questions about socio-demographic data and general food habits; the second part consisted of 12 questions and collected the frequency of consumption of various foods. Descriptive analysis was performed, bivariate and multivariate accordingly. Results: Both groups omitted mainly breakfast. Also, eating breakfast was a protective factor in the consumption of fast foods and soft drinks. Being on sixth year was a risk factor. Conclusion: Students of higher years consume fast foods and soft drinks more often, making it necessary to promote food education from the early years.
\end{abstract}

Key words: university students; fast food; food habits.

\section{INTRODUCCIÓN}

Los hábitos alimentarios adecuados son un aspecto clave para el desarrollo de una vida saludable. Sin embargo, a nivel mundial se observa una alta prevalencia de hábitos alimentarios inadecuados; siendo la prevalencia de consumo de comidas rápidas para el año 2011 de 17\% en Europa, 36\% en Asia Pacífico y $47 \%$ en las Américas (1).

Los hábitos alimentarios inadecuados son un importante factor de riesgo para el desarrollo de Enfermedades Crónicas No Transmisibles (ECNT) como obesidad, enfermedad coronaria, cáncer, diabetes, entre otras (2). Actualmente, la prevalencia de estas enfermedades está aumentando de manera alarmante a nivel mundial, de manera que anualmente las ENT causan 30 millones de muertes, 75\% de las cuales se producen en países de ingresos bajos y medios (3).

Los hábitos alimentarios se suelen adquirir tempranamente durante el desarrollo de la persona (2), influenciados por el aprendizaje de la persona en su contexto familiar, social y cultural (4). Sin embargo, algunas situaciones estresantes como el ingreso a la universidad generan una serie de cambios
Cristina Torres-Mallma (1) Claudia Trujillo-Valencia (1) Ana Lucía Urquiza-Díaz (1) Ronald Salazar-Rojas (1)

Alvaro Taype-Rondán (2)

(1) Facultad de Medicina Humana, Universidad de San Martín de Porres, Lima, Perú (2) CRONICAS Centro de Excelencia en Enfermedades Crónicas, Universidad Peruana Cayetano Heredia, Lima, Perú

Dirigir la correspondencia a: Cristina Torres-Mallma Dirección: Jr. Guardia Civil No 598 , Urb. Los Parrales de Surco, Santiago de Surco, Lima,

Teléfono: (+51) $980-035-587$

Correo electrónico: cristina_torres_95@hotmail.com

Este trabajo fue recibido el 21 de Noviembre de 2015 y aceptado para ser publicado el 5 de Marzo de 2016.

desfavorables en los estilos de vida, incluyendo la alimentación $(5,6)$. Por ello, implementar estrategias que prevengan tempranamente la adquisición o el reforzamiento de hábitos alimentarios inadecuados puede tener efectos significativos en la salud a corto y largo plazo (7). Sin embargo, lamentablemente la tendencia entre los estudiantes universitarios es la irregularidad en el consumo de comidas, y el consumo excesivo de comidas rápidas (8 -16).

En el Perú, el Instituto Nacional de Estadísticas e Informática (INEI), determinó que 33,8\% del total de habitantes de 15 y más años de edad presentaron sobrepeso, y 18,3\% obesidad el año 2013, siendo Lima Metropolitana la ciudad peruana que presentó el mayor porcentaje de sobrepeso y obesidad (17). De acuerdo al estudio del Centro Nacional de Alimentación y Nutrición del Perú realizado en el año 2010, existe una elevada prevalencia de consumo de comidas rápidas en todo el país, siendo esta prevalencia del 66\% en Lima Metropolitana (19).

Estudios realizados en estudiantes universitarios peruanos revelan un alto consumo de comidas rápidas que son frecuen- 
temente el alimento de principal en esta población (20-23). Sin embargo, poco se ha estudiado la tendencia de este consumo a lo largo de la vida universitaria. Otro tema poco estudiado ha sido la combinación de comida rápida y gaseosa, que según la Organización Mundial de Salud es frecuente y aumenta el riesgo de enfermedades crónicas (24).

El presente estudio se realizó con el objetivo de determinar los hábitos alimentarios de estudiantes de primer y sexto año de Medicina Humana, de una universidad privada de Lima (Perú), y para evaluar los factores asociados al consumo de gaseosas y comidas rápidas en dicha población.

\section{SUJETOS Y METODOS}

Diseño y lugar de estudio

Se realizó un estudio de tipo transversal analítico durante los meses de mayo y junio del año 2015 en los estudiantes de primer y sexto año de la Facultad de Medicina Humana de la Universidad de San Martin de Porres (FMH-USMP), Lima, Perú. La carrera de Medicina Humana en la FMH-USMP tuvo una duración de siete años: los tres primeros años se centraron en el estudio de ciencias básicas y se desarrollaron casi exclusivamente en el local de la FMH-USMP, mientras que los últimos 4 años se abocaron a la práctica clínica y se desarrollaron mayormente en diversas sedes hospitalarias.

\section{Sujetos}

La población de estudio estuvo conformada por 498 estudiantes de primer año y 322 estudiantes de sexto año, que representan al total de estudiantes matriculados en cada año. Para seleccionar a los estudiantes de primer año que entrarían al estudio, se realizó un muestreo por conglomerados utilizando el software Epidat 2.0, el cual seleccionó aleatoriamente 14 de los 30 grupos en los que los estudiantes estaban divididos para el curso de Lenguaje. De esta manera, se obtuvo una muestra de 226 estudiantes de primer año, de los cuales 219 (44,0\% del total de estudiantes de primer año) aceptaron participar en el estudio..

En el caso de sexto año, los estudiantes estuvieron distribuidos en diferentes hospitales. Por ello, se escogió el curso de Geriatría el cual posee mayor número de matriculados de los cuales 165 (51,2\% del total de estudiantes de sexto año) aceptaron participar en el estudio.

\section{PROCEDIMIENTOS}

Se encuestó a todos los estudiantes de primer año de los salones seleccionados antes del comienzo de sus clases, en coordinación con el docente encargado del curso. Antes de tomar las encuestas, se expuso los objetivos de la investigación. Cada cuestionario fue acompañado de un consentimiento informado. En el caso de menores de edad, se entregó junto al cuestionario un asentimiento informado y un consentimiento informado para los padres o apoderados.

En cuanto a los estudiantes de sexto año, fueron abordados durante uno de los exámenes teóricos del curso de Geriatría, al cual asistieron todos los estudiantes de dicho año. Los encuestadores explicaron de manera individual a cada estudiante los objetivos de la encuesta. De la misma manera, junto al cuestionario se entregó un consentimiento informado. En este caso todos los encuestados fueron mayores de edad. En todos los casos, los encuestadores estuvieron presentes durante todo el tiempo que tomó el Ilenado del cuestionario para aclarar las dudas que pudieron surgir. El tiempo de llenado en todos los casos fue en promedio de ocho minutos.

\section{INSTRUMENTO}

Los autores del presente estudio elaboraron un cuestionario autoaplicado que estuvo estructurado en 2 partes. La primera parte constó de 11 preguntas e incluyó los datos del estudiante (edad, sexo y año de estudio) y los hábitos alimentarios generales (número de comidas consumidas al día, frecuencia de desayuno, almuerzo y cena, y lugar donde suelen consumir sus alimentos). La segunda parte constó de 12 preguntas y recolectó la frecuencia de consumo de cada tipo de alimentos (grasas, comidas rápidas, frutas, verduras, alcohol, dulces, carnes, legumbres, bebidas energéticas, lácteas, café y cereales).

La encuesta fue elaborada en base a cuestionarios de hábitos alimentarios $(25,26)$. Debido a que los cuestionarios relacionados a Hábitos Alimentarios suelen ser complejos por la gran cantidad de preguntas, se resumió los principales ítems de acuerdo a los objetivos, siendo así una medición parcial de los hábitos alimentarios de los estudiantes. El instrumento fue validado por expertos: dos nutricionistas licenciadas en el área de nutrición y una en metodología. Se realizaron las respectivas correcciones teniendo en cuenta el objetivo del estudio, la formación académica de los jueces, explicitando las dimensiones e indicadores que mide cada variable, de esta manera evaluando la suficiencia y pertinencia del ítem. Finalmente, se hizo el diseño del cuestionario teniendo en cuenta los objetivos del estudio. Cabe resaltar que todo este proceso se realizó en conjunto con los jueces expertos.

\section{ANÁLISIS DE DATOS}

Una vez recolectados los cuestionarios, fueron digitados doblemente en el programa Microsoft Excel 2010. Posteriormente la base de datos fue exportada al software estadístico STATA v13.0 donde se procesó la información. Las variables fueron presentadas mediante frecuencias absolutas, frecuencias relativas, medidas de tendencia central y de dispersión. Se realizó un análisis bivariado mediante la prueba exacta de Fisher y la prueba de $\mathrm{t}$ de Student según correspondía. Se realizó un análisis de múltiples variables para evaluar los factores asociados a tener un consumo frecuente de comida rápida y gaseosas, utilizando la regresión de Poissón cruda y múltiple con varianzas robustas. El $\mathrm{p}<0.05$ fue considerado como punto de corte para el valor significativo.

Para el análisis de los factores asociados al consumo frecuente de comida rápida y bebidas gaseosas, se elaboró una variable compuesta, de tal manera que los casos positivos fueron aquellos que consumían comida rápida y bebidas gaseosas frecuentemente (al menos tres veces por semana), y los casos negativos fueron aquellos que no consumían ni comida rápida ni bebidas gaseosas frecuentemente. Se decidió evaluar esta variable debido a que representa a las dos opciones de comida chatarra que más se consumen, según lo encontrado en nuestro estudio.

\section{RESULTADOS}

Se obtuvieron un total de 384 encuestas: 218 (56,8\%) de estudiantes de primer año y $166(43,2 \%)$ de estudiantes de sexto año. El 57,3\% de los estudiantes de primer año y 53,0\% de los de sexto año fueron del sexo femenino. En cuanto al lugar donde se consume generalmente los alimentos se encontró que los estudiantes de primer año solían comer en su casa en mayor porcentaje que los de sexto año (57,8\% versus $50,0 \%)$, aunque esta diferencia no fue significativa. El porcentaje de estudiantes que consumían tres o más comidas al día fue $11,5 \%$ en primer año y $30,1 \%$ en sexto año $(p<0,001)$. En cuanto a 


\section{TABLA 1}

Características de los estudiantes encuestados $(\mathrm{N}=384)$.

\begin{tabular}{|c|c|c|c|}
\hline & $\begin{array}{c}\text { 1er año } \\
(\mathrm{N}=218)\end{array}$ & $\begin{array}{l}\text { 6to año } \\
(N=166)\end{array}$ & $\mathrm{p}$ valor \\
\hline Edad & $18.0 \pm 1.2$ & $23.0 \pm 1.2$ & \\
\hline \multicolumn{4}{|l|}{ Sexo } \\
\hline Femenino & $125(57,3)$ & $88(53,0)$ & \multirow[t]{2}{*}{0.398} \\
\hline Masculino & $93(42,7)$ & $78(47,0)$ & \\
\hline \multicolumn{4}{|l|}{ Lugar donde generalmente consume sus alimentos } \\
\hline Casa & $126(57,8)$ & $83(50,0)$ & \multirow[t]{2}{*}{0.247} \\
\hline Fuera de casa & $92(42,2)$ & $83(50,0)$ & \\
\hline \multicolumn{4}{|l|}{ Cuantas comidas consume al día } \\
\hline Ninguna & $5(2,3)$ & $2(1,2)$ & \multirow[t]{4}{*}{$<0.001$} \\
\hline Una comida al día & $44(20,2)$ & $24(14,5)$ & \\
\hline Dos comidas al día & $144(66,1)$ & $90(54,2)$ & \\
\hline Tres o más comidas al día & $25(11,5)$ & $50(30,1)$ & \\
\hline \multicolumn{4}{|l|}{ Malos hábitos alimenticios } \\
\hline Consumo frecuente de comida rápida y de gaseosas & $29(13,3)$ & $31(18,7)$ & \multirow[t]{3}{*}{0.171} \\
\hline Consumo frecuente de comida rápida o de gaseosas & $74(33,9)$ & $62(37,4)$ & \\
\hline No presentó consumo frecuente de comida rápida ni de gaseosas & $115(52,8)$ & $73(44,6)$ & \\
\hline
\end{tabular}

\section{FIGURA 1}

Frecuencia de consumo de comidas en los encuestados, por año de estudios.

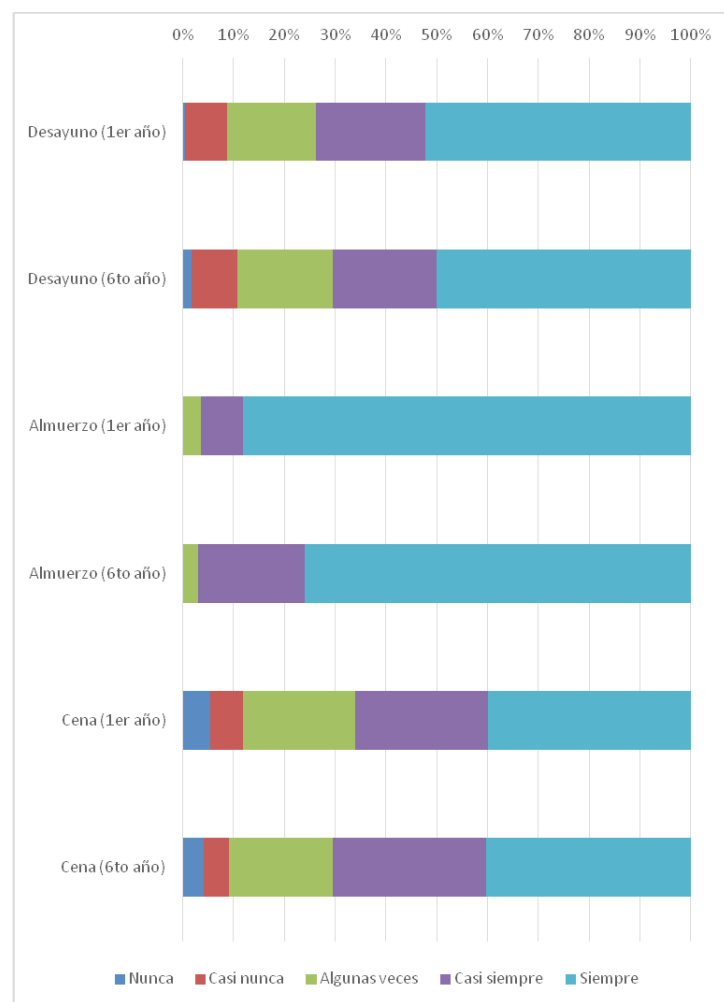


los hábitos alimentarios, 13,3\% de los estudiantes de primer año y $18,7 \%$ de los estudiantes de sexto año presentaban un consumo de comida rápida y gaseosas de al menos tres veces por semana (tabla 1).

En cuanto a la frecuencia de comidas, el porcentaje de estudiantes que siempre consumen desayuno, almuerzo y cena fue $51,3 \%, 82,8 \%$ y $40,1 \%$ respectivamente. Al comparar estas variables con el año de estudios, se encontró diferencia significativa en cuanto al almuerzo, que fue consumido por el $88,1 \%$ de los estudiantes de primer año y 75,9\% de los estudiantes de sexto año $(p=0.0017)$. El consumo del resto de alimentos no estuvo asociado al año de estudios (figuras 1, 2 y 3).

Al evaluar el consumo de los diferentes tipos de alimentos entre estudiantes de primero y sexto año, 28,9\% consumía comida rápida y $37,8 \%$ consumía gaseosas frecuentemente. Al comparar el consumo frecuente de distintos alimentos con el año de estudios, se encontró diferencias significativas en cuanto al consumo de: leche $(p=0.023)$, café $(p=0.028)$ y alcohol $(p=0.000)$. (tabla 2$)$.

En cuanto a los factores asociados al consumo frecuente (al menos tres veces por semana) de comida rápida y bebidas gaseosas, el análisis de múltiples variables determinó los siguientes: cursar el sexto año de estudios ( $R P=1,55$; IC95\%: $1.02-2.35)$, y tomar desayuno siempre ( $R P=0,39 ; \quad I C 95 \%$ : $0,23-0,64)$. No se encontró asociación significativa con las otras variables (tabla 3 ).

\section{DISCUSIÓN}

Actualmente se recomienda el consumo de tres o más alimentos al día para mantener los niveles de glucosa necesarios para realizar las actividades diarias (27). Sin embargo, sólo 11,5\% de los estudiantes de primer año y el 30,1\% de los de sexto año consumen por lo general tres o más comidas al día. Estas cifras bajas requieren estudios posteriores, aunque posiblemente se deban a un estilo de vida desordenado, producto de la exigencia académica y la falta de educación alimentaria. Bajo esta hipótesis, los estudiantes de primer año estarían más expuestos, probablemente porque tienen que adaptarse bruscamente a un nuevo estilo de vida, descuidando su alimentación (28). Aunque se ha observado que esta adaptación depende del colegio de procedencia y de las herramientas que tengan los estudiantes para afrontar los cambios (29). De cualquier manera, estos resultados reflejan la necesidad de que las universidades cuiden el bienestar físico y mental del estudiante durante los primeros ciclos, brindándole las herramientas necesarias para afrontar los cambios sin caer en malos hábitos ni trastornos de la conducta alimentaria $(30,31)$.

El $15,6 \%$ de los encuestados consumían comida rápida y gaseosas frecuentemente (al menos tres veces por semana), lo cual guarda similitud con estudios realizados en estudiantes universitarios en otros países $(32,33)$, y que refleja un problema de malos hábitos alimentarios que debe ser abordado por las

\section{FIGURA 2}

Frecuencia de consumo de tipos de alimentos en estudiantes de primer año.

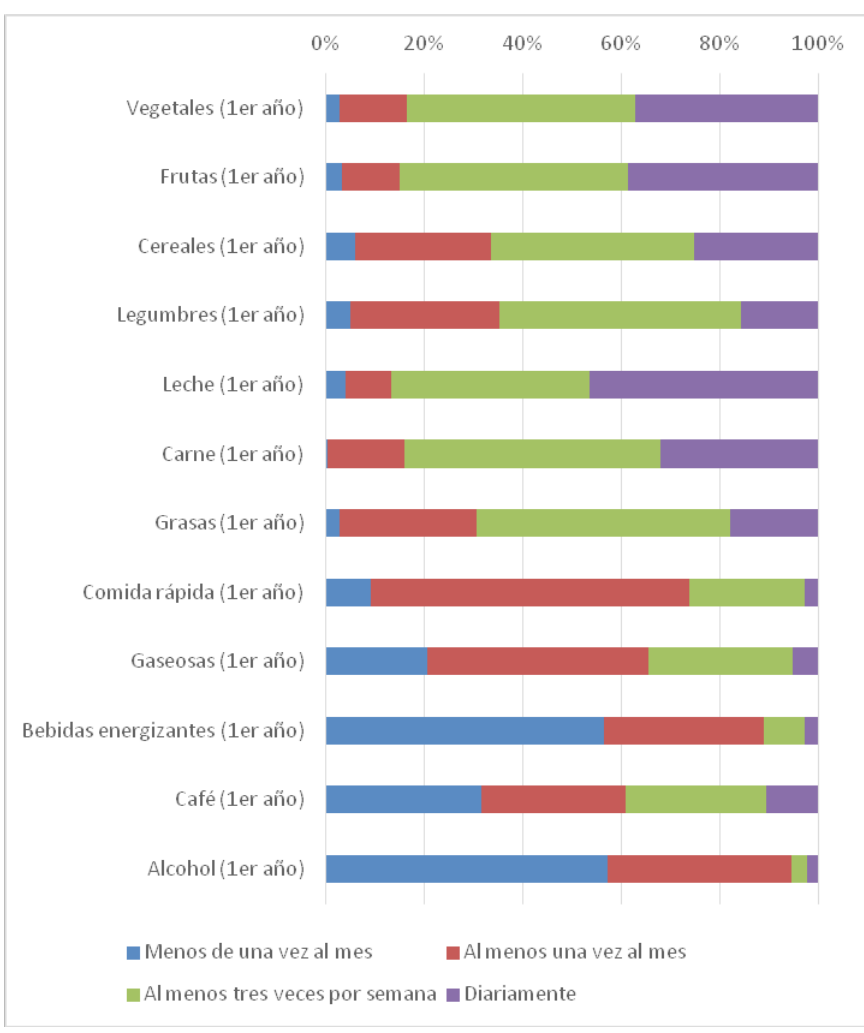


universidades.

Ante esta problemática, las "universidades saludables" deben implementar programas nutricionales universitarios que sustituyan las comidas rápidas por alimentos saludables como frutas y verduras en las cafeterías universitarias (34), así como cursos de capacitación sobre educación nutricional para fomentar conductas saludables (35), trabajar el entorno familiar mediante charlas y/o asambleas magistrales. (36), e incluso trabajar con estudiantes en riesgo mediante consultas personales que logren diseñar un régimen alimenticio personalizado de acuerdo a sus necesidades particulares para alcanzar un cambio sostenible en sus hábitos inadecuados (35).

Los estudiantes de sexto año tuvieron un mayor riesgo de este consumo, probablemente debido a que pasan la mayor parte de su tiempo en diversas sedes hospitalarias, donde la comida rápida y las gaseosas sean los alimentos y las bebidas más accesibles. Otra explicación sería que con el pasar de los años estos estudiantes adquieren más libertad y corren el riesgo de desarrollar malos hábitos alimentarios. Por lo cual parece lógico prevenir actuando desde los primeros años de estudio $(38,39)$.

Se encontró que las comidas más consumidas en los de sexto año fue alcohol y café, mientras que, en los de primer año fue la leche. Ello es similar con los resultados de un estudio realizado en Colombia con estudiantes de odontología (40), difiere de otros estudios en los que la leche es un alimento relativamente poco consumido en estudiantes universitarios (41). En un estudio realizado en estudiantes de medicina de una universidad de Croacia (26), los alumnos de sexto año también consumían significativamente más café que aquellos de primer año; sin embargo en el caso de leche y alcohol no se encontró diferencia significativa.

También se encontró que tomar desayuno fue un factor protector para el consumo frecuente de comida rápida y bebidas gaseosas. Este hallazgo podría deberse a que los estudiantes que no toman desayuno optan eventualmente por consumir aquello que tengan más a la mano en su centro de estudios (42). Para combatir esto, se ha diseñado la estrategia de desayunos universitarios (43), capacitando a las concesionarias para brindar desayunos de calidad, como se ha realizado en México (44), España y Ecuador $(45,46)$. En el Perú, una universidad en Piura facilita que los estudiantes puedan armar su desayuno con alimentos que se venden en la cafetería (47). Por otro lado, un estudio realizado en vendedores de desayuno ambulantes en Lima Metropolitana, reveló que el $85 \%$ de estos no contaron con un adecuado nivel de conocimientos sobre alimentación, lo que conlleva a que no ofrezcan desayunos de buena calidad (48), situación que podría existir en las concesionarias universitarias, y que merece ser estudiada.

El desayuno es la comida principal del día, ya que el organismo obtiene la mayor fuente de energía para realizar las

\section{FIGURA 3}

Frecuencia de consumo de tipos de alimentos en en estudiantes de sexto año.

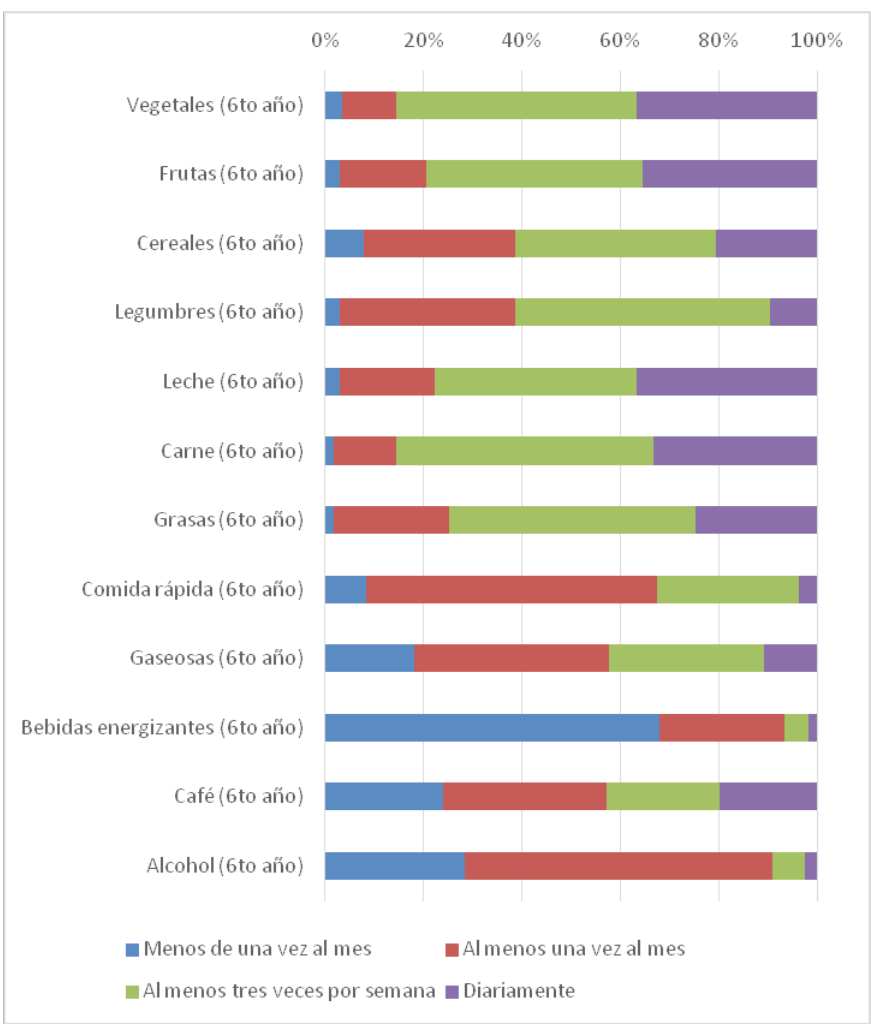


actividades cotidianas (49), por lo que su ausencia, repercute negativamente en el estudiante, dificultando su rendimiento académico (50). Existen diversos estudios que han demostrado que la omisión del desayuno en la adolescencia y juventud está asociado a desarrollar una ECNT como la diabetes, obesidad y enfermedades coronarias en la edad adulta (51-53), y se ha evidenciado que tomar un desayuno saludable basado en fibra y frutas, reduce el riesgo de sobrepeso (54).

Sin embargo, sólo $51.3 \%$ de los participantes afirmaron tomar desayuno siempre. Resultado similar al obtenido en un estudio realizado en estudiantes universitarios del Sur de Chile (41). No obstante, esta cifra resulta baja en comparación a estudios de Polonia (55), Buenos Aires (56), España (57) y Túnez (58). Esta baja cifra puede deberse a factores como el horario de clases, sobrecarga de estudios, la calidad de sueño y otros aspectos psicológicos y socioeconómicos $(59,60)$. Además, es probable que la población estudiada presente menor educación alimentaria, lo cual requiere la ejecución de programas nutricionales (enfatizando la selección, compra, preparación de los alimentos) a nivel nacional y particularmente a nivel de las universidades, siendo la educación alimentaria una estrategia de prevención de primer nivel de las ECNT (61).

Es necesario mencionar algunas limitaciones metodológicas del presente estudio: la tasa de rechazo en la población de sexto año fue alta, probablemente debido a su ajustado horario. También existe el riesgo de que los participantes menos preocupados por sus hábitos alimentarios presenten mayor sesgo de memoria. Además, hay que tomar en cuenta que estos resultados provienen de una universidad particular, con estudiantes que comparten ciertas características, por lo cual no podrán extrapolarse a todos los estudiantes de Medicina Humana del Perú, siendo necesario realizar estudios multicéntricos para ello. Sin embargo, este estudio con pocos antecedentes en el Perú nos alerta sobre una alta prevalencia de malos hábitos alimentarios, y la necesidad de intervenir en factores asociados puntuales.

En conclusión, el presente estudio evidenció inadecuados hábitos alimentarios en estudiantes de medicina de la Universidad San Martín de Porres, como la omisión de desayuno y el consumo de comidas rápidas. Asimismo, se encontró que cursar sexto año puede ser un factor de riesgo, y que tomar desayuno podría ser un factor protector al consumo frecuente de comidas rápidas y gaseosas. Es indispensable la intervención en las universidades para promocionar una educación alimentaria desde los primeros años, así como la aplicación de estrategias en los estudiantes y concesionarias universitarias en favor de un desayuno saludable.

\section{RESUMEN}

Objetivo: Determinar los hábitos alimentarios y evaluar los factores asociados al consumo de gaseosas y comida rápida. Sujetos y métodos: Estudio transversal analítico, realizado

\section{TABLA 2}

Frecuencia de consumo de tipos de alimentos en los encuestados, por año de estudios.

\begin{tabular}{|c|c|c|c|c|c|}
\hline & $\begin{array}{c}\text { Menos de una } \\
\text { vez al mes }\end{array}$ & $\begin{array}{l}\text { Al menos una } \\
\text { vez al mes }\end{array}$ & $\begin{array}{c}\text { Al menos tres } \\
\text { veces por semana }\end{array}$ & Diariamente & $\mathrm{p}$ valor \\
\hline Vegetales (1er año) N=218 & $6(2,8)$ & $30(13,76)$ & $101(46,3)$ & $81(37.2)$ & \\
\hline Vegetales (6to año) N=116 & $6(3,6)$ & $18(10,84)$ & $81(48,8)$ & $61(36,7)$ & 0,803 \\
\hline Frutas (1er año) & $7(3,2)$ & $26(11,93)$ & $101(46,3)$ & $84(38,5)$ & \\
\hline Frutas (6to año) & $53,0)$ & $29(17,47)$ & $73(44,0)$ & $59(35,5)$ & 0,498 \\
\hline Cereales (1er año) & $13(6,0)$ & $60(27,52)$ & $90(41,3)$ & $55(25,2)$ & \\
\hline Cereales (6to año) & $13(7,8)$ & $51(30,72)$ & $68(41,0)$ & $34(20,5)$ & 0,628 \\
\hline Legumbres (1er año) & $11(5,0)$ & $66(30,28)$ & $107(49,1)$ & $34(15,6)$ & \\
\hline Legumbres (6to año) & $5(3,0)$ & $59(35,54)$ & $86(51,8)$ & $16(9,6)$ & 0,217 \\
\hline Leche (1er año) & $94,1)$ & $20(9,17)$ & $88(40,4)$ & $101(46,3)$ & \\
\hline Leche (6to año) & $5(3,0)$ & $32(19,28)$ & $68(41,0)$ & $61(36,7)$ & 0,023 \\
\hline Carne (1er año) & $1(0,5)$ & $34(15,60)$ & $113(51,8)$ & $70(32,1)$ & \\
\hline Carne (6to año) & $3(1,8)$ & $21(12,65)$ & $87(52,4)$ & $55(33,1)$ & 0,522 \\
\hline Grasas (1er año) & $6(2,8)$ & $61(27,98)$ & $112(51,4)$ & $39(17,9)$ & \\
\hline Grasas (6to año) & $3(1,8)$ & $39(23,49)$ & $83(50,0)$ & $41(24,7)$ & 0,359 \\
\hline Comida rápida (1er año) & $20(9,2)$ & $141(64,68)$ & $51(23,4)$ & $62,8)$ & \\
\hline Comida rápida (6to año) & $14(8,4)$ & $98(59,04)$ & $48(28,9)$ & $6(3,6)$ & 0,598 \\
\hline Gaseosas (1er año) & $4520,6)$ & $98(44,95)$ & $64(29,4)$ & $11(5,0)$ & \\
\hline Gaseosas (6to año) & $30(18,1)$ & $66(39,76)$ & $52(31,3)$ & $18(10,8)$ & 0,212 \\
\hline Bebidas energizantes (1er año) & $123(56,4)$ & $71(32,57)$ & $188,3)$ & $6(2,8)$ & \\
\hline Bebidas energizantes (6to año) & $113(68,1)$ & $42(25,30)$ & $8(4,8)$ & $3(1,8)$ & 0,123 \\
\hline Café (1er año) & $69(31,7)$ & $64(29,36)$ & $62(28,4)$ & $23(10,6)$ & \\
\hline Café (6to año) & $40(24,1)$ & $55(33,13)$ & $38(22,9)$ & $33(19,9)$ & 0,028 \\
\hline Alcohol (1er año) & $125(57,3)$ & $81(37,16)$ & $7(3,2)$ & $5(2,3)$ & \\
\hline Alcohol (6to año) & $47(28,3)$ & $104(62,65)$ & $11(6,6)$ & $4(2,4)$ & 0,000 \\
\hline
\end{tabular}


en estudiantes de primer $(n=269)$ y sexto año $(n=165)$ de la Facultad de Medicina de la Universidad San Martín de Porres, de Lima, Perú. Se diseñó y aplicó un cuestionario estructurado en dos partes: la primera parte constó de 11 preguntas que incluyó los datos sociodemográficos y hábitos alimentarios generales; la segunda parte constó de 12 preguntas y recolectó la frecuencia de consumo de varios alimentos. Se realizó el análisis descriptivo, bivariado y multivariado correspondiente. Resultados: Ambos grupos omitieron principalmente el desayuno. Asimismo, tomar desayuno fue un factor protector del consumo de comidas rápidas y gaseosas, y cursar sexto año como un factor de riesgo. Conclusión: Los estudiantes de años superiores consumen comidas rápidas y gaseosas con mayor frecuencia, por lo que resulta necesario promover la educación alimentaria desde los primeros años.

Palabras clave: estudiantes universitarios; comida rápida; hábitos alimentarios.

\section{BIBLIOGRAFÍA}

1. Moneo J, Sirgado M, Lamas Á. Fast Food Consumption. [Internet]. Recovered from: http://www.abc.es/gestordocumental/uploads/Sociedad/comida-rapida.pdf

2. Expert Consultation on Diet, Nutrition, and the Prevention of Chronic Diseases, FAO. Geneva: World Health Organization; 2003. p 149.

3. OMS. Non Communicable Diseases [Internet]. Recovered from: http://www.who.int/mediacentre/factsheets/ fs355/es/

4. Delavari M, Sønderlund AL, Mellor D, Mohebbi M, Swinburn B. [Migration, Acculturation and Environment: Determinants of Obesity among Iranian Migrants in Australia]. Int J Environ Res Public Health. 2015;12(2):1083-98.

5. Podstawski R, Choszcz D, KlimczakJ, Kolankowska E, Zurek $P$. [Habits and attitudes of first-year female students at
Warmia and Mazury University: a call for implementing health education programme at universities]. Cent Eur J Public Health. 2014;22(4):229-38.

6. Cruwys T, Bevelander KE, Hermans RCJ. [Social modeling of eating: a review of when and why social influence affects food intake and choice. Appetite]. 2015;86:3-18.

7. Organization WH, others. Global Strategy on Diet, Physical Activity and Health. 2004. [Internet]. Recovered from: http://apps.who.int/iris/handle/10665/43037

8. Sáenz S, González F, Díaz S. [Habits and Eating Disorders associated with socio-demographic factors, physical and behavioral in University of Cartagena, Colombia]. Rev Clín Med Familia. 2011;4(3):193-204.

9. Espinoza O, Rodríguez R, Gálvez C, MacMillan $K$. [Eating Habits and Physical Activity in College Students]. Rev Chil Nutr. 2011;38(4):458-65.

10. Rodríguez R, Palma L, Romo B, Escobar B, Aragú G, Espinoza $O$, et al. [Eating habits, physical activity and socioeconomic status in university students in Chile]. Nutr Hosp. 2013;28(2):447-55.

11. Pelletier J, Laska M. [Campus food and beverage purchases are associated with indicators of diet quality in college students living off campus]. AJHP. 2013;28(2):80-7.

12. Paeratakul S, Ferdinand D, Champagne C, Ryan D, Bray G. [Fast-food consumption among US adults and children: dietary and nutrient intake profile]. J Am Diet Assoc. 2003;103(10):1332-8.

13. Niemeier H, Raynor H, Lloyd-Richardson E, Rogers L, Wing $R$. [Fast food consumption and breakfast skipping: predictors of weight gain from adolescence to adulthood in a nationally representative sample]. J Adolesc Health Off Publ Soc Adolesc Med. 2006;39(6):842-9.

14. Correa P, Sifontes M de, Zulay M, Bauce $G$, Cueva E, et al. [Patterns and eating habits: eat reflection of what young

\section{TABLA 3}

Factores asociados al consumo frecuente de comida rápida y bebidas gaseosas.

\begin{tabular}{|c|c|c|c|c|c|}
\hline & & \multicolumn{2}{|c|}{$\begin{array}{l}\text { Consumo frecuente (al menos } \\
\text { tres veces por semana) de } \\
\text { omida rápida y bebidas gaseosas }\end{array}$} & $\begin{array}{l}\text { Crudo } \\
\text { RP (IC95\%) }\end{array}$ & $\begin{array}{l}\text { Multivariado } \\
\text { RP (IC95\%) }\end{array}$ \\
\hline \multirow[t]{2}{*}{ Edad* $^{*}$} & Mitad inferior & $129(72,9)$ & $48(27,1)$ & Ref & Ref \\
\hline & Mitad superior & $59(83,1)$ & $12(16,9)$ & $0,62(0,35-1,10)$ & $0,61(0,34-1,06)$ \\
\hline Sexo & Masculino & $87(76,3)$ & $27(23,7)$ & $0,96(0,62-1,50)$ & $1,08(0,71-1,65)$ \\
\hline \multirow[t]{2}{*}{ Año } & 1er año & $115(79,9)$ & $29(20,1)$ & Ref & Ref \\
\hline & 6to año & $73(70,2)$ & $31(29,8)$ & $1,48(0,95-2,30)$ & $1,55(1,02-2,35)$ \\
\hline \multirow[t]{2}{*}{ Dónde toma sus alimentos } & Fuera de su casa & $85(73,9)$ & $30(26,1)$ & Ref & Ref \\
\hline & En su casa & $103(77,4)$ & $30(22,6)$ & $0,86(0,56-1,34)$ & $1,0(0,64-1,54)$ \\
\hline \multirow[t]{2}{*}{ Toma cena } & No siempre & $112(73,2)$ & $41(26,8)$ & Ref & Ref \\
\hline & siempre & $76(80,0)$ & $19(20,0)$ & $0,75(0,46-1,21)$ & $0,83(0,52-1,24)$ \\
\hline
\end{tabular}


ucevistas]. Rev Fac Med Caracas. 2009;67-74.

15. Chourdakis M, Tzellos T, Papazisis G, Toulis K, Kouvelas $D$. [Eating habits, health attitudes and obesity indices among medical students in northern Greece]. Appetite. 2010;55(3):722-5

16. De Piero A, Bassett $N$, Rossi A, Sammán $N$. [Trends in food consumption of university students]. Nutr Hosp. 2015;31(4):1824-31.

17. INEI. Noncommunicable Diseases and Communicable Diseases in Peru, 2013. Lima; 2014 [Internet]. Recovered from: http://www.inei.gob.pe/media/MenuRecursivo/ publicaciones_digitales/Est/Lib1152/libro.pdf

18. OMS. World Health Organization - NCD Country Profiles, 2014 [Internet]. Recovered from: http://www. who.int/ $n m h /$ countries/per_es.pdf?ua $=1$

19. Haydee G. National Nutrition Survey Indicators, Biochemicals, Socioeconomic and Cultural Related Chronic Diseases Degenerative 2006. [Internet]. Recovered from: http:// www.minsa.gob.pe/portada/Especiales/2007/nutricion/ publicaciones/informe_final_enin.pdf

20. Sevillano-García M, Sotomayor-Baca A. [Food Advertising and Consumption by Students in Huánuco, Peru]. Comunicar. 2012;20(39):177-84.

21. Ferro $R$, Maguiña $V$. [Relationship between eating habits and body mass index in a public university students as study area]. 2012 [Internet]. Recovered from: http://cybertesis. unmsm.edu.pe/handle/cybertesis/1123

22. Sovero $M$, Elida $M$. [Lifestyles of nursing students of the National University of San Marcos, Perú] 2006. [Internet]. Recovered from: http://cybertesis.unmsm.edu.pe/handle/ cybertesis $/ 498$

23. Muchotrigo M. [Construction of an Instrument on healthy lifestyles in College Students]. Rev Psicol. 2015;9(1):8-20.

24. Rodríguez IT, Ballart JF, Pastor GC, Jordà EB, Val VA. [Validation of a short questionnaire on frequency of dietary intake: reproducibility and validity]. Nutr Hosp. 2008;23(3):242-52.

25. Nola I, Jelinić J, Matanić D, Pucarin-Cvetković J, Bergman Marković $B$, Senta A. [Differences in eating and lifestyle habits between first- and sixth-year medical students from Zagreb]. Coll Antropol. 2010;34(4):1289-94.

26. Llauradó $E$, Albar A, Giralt $M$, Solà $R$, Evans $C$. [The effect of snacking and eating frequency on dietary quality in British adolescents]. Eur J Nutr. 2016;55(4):1789-97.

27. Celis J, Araujo MB, Cabrera D, Cabrera M, Alarcón W, Monge E. [Academic Anxiety and Stress in Students of Human Medicine of the First and Sixth Year]. An Fac Med. 2014;62(1):25-30.

28. Quiroz F, Narbajo Y, Chávez G. [Emotional intelligence in students of the first cycle of a private university in Lima Metropolitana]. Rev Investi Psicol; 2003;6(2); 153-66.

29. Jáuregui Lobera I, Polo I, González M, Millán M. [Perception of obesity in university students and in patients with eating disorders]. Nutr Hosp. 2008;23(3):226-33.

30. Castaño-Castrillón J, Giraldo D, Guevara J, Losada D, Meza L, Narváez D, et al. [Prevalence of risk of eating disorders in a female population of high school students, Manizales, Colombia], 2011. Rev Colomb Obstet Ginecol. 2012;63(1):46-56.

31. Becerra-Bulla F, Pinzón-Villate G, Vargas-Zárate M. [Medical students nutritional status and food consumption, in UNAL-Bogotá 2010-2011]. Rev Fac Med. 2012;60: 3-12.

32. Martinez C, Kriscovich J, Jorge O, Herdt A, Lopez E, Ibarra $R$, De Bonis $G$, Correa L. [Prevalence of cardiovascular risk factors in medical students. United Nations Information Center for Argentina and Uruguay: Chair of Semiotics and Pathophysiology, Faculty of Medicine. Northeastern University : scientific and technological Communications] 2000.

33. Freire $M$, Bou $R$, Cofrades $S$, Jiménez Colmenero $F$, et al. [Encapsulation of vitamin B2 in double emulsions: Influence of the type of oil]. Nutr Clin Diet Hosp. 2014;34(258).

34. Mardones H, Olivares C, Araneda F, Gómez F. [Stages of change related to the consumption of fruits and vegetables, physical activity and weight control in Chilean university students]. Arch Latinoam Nutr. 2009;59(3):304-9.

35. Karlen G, Masino $M$, Fortino $M$, Martinelli $M$. [Breakfast consumption among college students: habit, nutritional quality and its relationship with body mass index]. Dieta. 2011;29(137):23-30.

36. Martínez $M$, Hernández $M$, Ojeda $M$, Mena $R$, Alegre $A$, Alfonso J. [Development of a program of nutritional education and valuation of the change of healthful nourishing habits in a population of students of Obligatory Secondary Education]. Nutr Hosp. 2009;24(4):504-10.

37. Consumers International. Junk Food Generation : Toolkit for national campaigns [Internet]. Recovered from: http:// www.consumersinternational.org/media/573539/generacioncomidachatarraconjuntodeherramientas.pdf

38. Baldinger N, Krebs A, Müller R, Aeberli I. [Swiss children consuming breakfast regularly have better motor functional skills and are less overweight than breakfast skippers]. J Am Coll Nutr. 2012;31(2):87-93.

39. Ibáñez E, Thomas Y, Bicenty A, Barrera J, Martínez J, Gerena $R$. [Changes in eating habits dental students of the University Foundation San Martin de Bogota, Colombia]. NOVA [Internet]. Recovered from: http://www. unicolmayor.edu. co/publicaciones/index.php/nova/article/view/103

40. Rivera Barragán M del R. [Eating habits in students of the University Juárez Autónoma de Tabasco]. Rev Cuba Salud Pública. 2006;32(3):0-7.

41. Lachat $C$, Nago $E$, Verstraeten $R$, Roberfroid D, Van Camp $J$, Kolsteren P. [Eating out of home and its association with dietary intake: a systematic review of the evidence]. Obes Rev Off J Int Assoc Study Obes. 2012;13(4):329-46.

42. Escalona Y, Bauce G, Mata E. [Vision of cafes and restaurants - Central University of Venezuela]. Tribuna del Investigador. 2000;7(2)

43. Georgina L, Esteban J, Camacho M, Ibarra Espinosa, Regional L, López M, et al. [Eating habits of college students]. Rev Digital Universitaria 2013;14(11).

44. Combos and breakfast for college [Internet]. El Universo. Recovered from: http://www.eluniverso.com/ noticias/2014/11/20/nota/4245181/combos-desayunosuniversitarios

45. University of Alcalá (UAH). Canteens and cafeterias [Internet]. Recovered from: https://www.uah.es/servicios/ servicios_ayudas_prestaciones/cafeterias.shtm

46. Breakfast Kings. University of Piura [Internet]. Recovered from: http://udep.edu.pe/hoy/2013/desayuno-de-reyes/

47. Salazar J, Vera R. [Nutritional quality of breakfast offered in ambulatory sales positions and level of knowledge about feeding in Lima]. Fac Med. Renut 2011;5(15): 788-96.

48. Anzman-Frasca S, Djang HC, Halmo MM, Dolan PR, Economos CD. [Estimating impacts of a breakfast in the classroom program on school outcomes]. JAMA Pediatr. 2015;169(1):71-7.

49. Rampersaud G, Pereira M, Girard B, Adams J, Metzl J. 
[Breakfast Habits, Nutritional Status, Body Weight, and Academic Performance in Children and Adolescents]. J Am Diet Assoc. 2005;105(5):743-60.

50. Maria Wennberg, Per E Gustafsson, Patrik Wennberg and Anne Hammarström (2015). [Poor breakfast habits in adolescence predict the metabolic syndrome in adulthood. Public Health Nutrition], 18, pp. 122-129.

51. Wennberg M, Gustafsson PE, Wennberg $P$, Hammarström A. [Irregular eating of meals in adolescence and the metabolic syndrome in adulthood: results from a 27-year prospective cohort]. Public Health Nutr. 2015;1-7.

52. Chung S-J, Lee Y, Lee S, Choi K. [Breakfast skipping and breakfast type are associated with daily nutrient intakes and metabolic syndrome in Korean adults]. Nutr Res Pract. 2015;9(3):288-95.

53. Nolan L. [Noncommunicable Diseases and Youth: A Critical Window Of Opportunity for Latin America and the Caribbean]. 2013 [Internet]; Recovered from: http://www. prb.org/pdf13/noncommunicable-diseases-latin-americayouth-policybrief.pdf

54. Likus W, Milka D, Bajor G, Jachacz-Łopata M, Dorzak $B$. [Dietary habits and physical activity in students from the Medical University of Silesia in Poland]. Rocz Państw Zakładu Hig. 2013;64(4):317-24.
55. Karlen G, Masino MV, Fortino MA, Martinelli $M$. [Breakfast consumption among college students : habit, nutritional quality and its relationship with body mass index]. Diaeta. 2011;29(137):23-30.

56. Montero Bravo A, Ubeda Martín N, García González A. [Evaluation of dietary habits of a population of university students in relation with their nutritional knowledge]. Nutr Hosp. 2006;21(4):466-73.

57. Cervera Burriel F, Serrano Urrea R, Daouas $T$, Delicado Soria A, García Meseguer MJ. [Food habits and nutritional assessment in a tunisian university population]. Nutr Hosp. 2014;30(6):1350-8.

58. Sun J, Yi H, Liu Z, Wu Y, Bian J, Wu Y, et al. [Factors associated with skipping breakfast among Inner Mongolia medical students in China]. BMC Public Health. 2013;13:42.

59. Ganasegeran K, Al-Dubai S, Qureshi A, Al-abed A, AM R, Aljunid $S$. [Social and psychological factors affecting eating habits among university students in a Malaysian medical school: a cross-sectional study]. Nutr J. 2012;11:48.

60. Olivares S, Jara B. Nutrition education as a strategy to strength family farmers and benefit the food and nutrition of the population | Global Forum on Food Security and Nutrition [Internet]. Recovered from: http://www.fao. org/fsnforum/es/forum/discussions/nutrition-education. 